\title{
Electron Transport In One-Dimensional Magnetic Superlattices
}

\author{
ZHEN-LI JI* and D. W. L. SPRUNG \\ Department of Physics and Astronomy, McMaster University, Hamilton, Ontario, Canada L8S 4M1

\begin{abstract}
Electron transport properties of quantum wires in the presence of a periodically modulated magnetic field are investigated. For a short modulated wire, we find dips in conductance just below each mode threshold. The conductance dips are quite robust at low temperature. Increasing the number of periods of magnetic modulation can lead to the formation of minibands and gaps. The differences between the one dimensional (1D) electric superlattice and 1D magnetic superlattice are discussed. We also consider the spatial distributions of currents, which show dramatic differences between the magnetic superlattices and electric ones.
\end{abstract}

Keywords: Two-dimensional electron gas, magnetotransport, nanostructure, quantum wire, superlattice, miniband

Recently, much attention has been paid to electron transport in magnetic fields that are inhomogeneous on the nanometer scale. Experimentally, such magnetic modulation can be realized by laying metallic strips on the surface of the heterostructure, consisting of either superconducting or ferromagnetic material $[1,2,3]$, or by varying the topography of a non-planar two-dimensional electron gas (2DEG) grown at a GaAs/AlGaAs heterojunction [4].

In this paper we point out that the experimental study of a new class of semiconductor nanostructures, the 1D magnetic superlattices, is now within reach and we present theoretical predictions of their transport properties.
We consider a model of a two-dimensional ballistic quantum wire shown schematically in Figure 1. This quantum wire has infinite length in the $x$ direction and is confined laterally $-W /$ $2<y<W / 2$ by hard wall potentials. Inside the channel the potential is taken to be zero. Our system consists of a finite magnetic superlattice and two straight leads. A uniform background field $B_{0}$ is applied to the entire sample, while a finite section of length $L=\mathrm{Na}$ is subject to an additional periodically varying magnetic field with amplitude $B_{m}$ perpendicular to the plane $[5,6]$.

For a quantum wire without modulation, it is well-known that conductance is quantized in units

*Corresponding author: Tel.: (905) 525-9140 Ext. 23181 Email: Zheji@physun.physics.mcmaster.ca Fax: (905) 546-1252. 


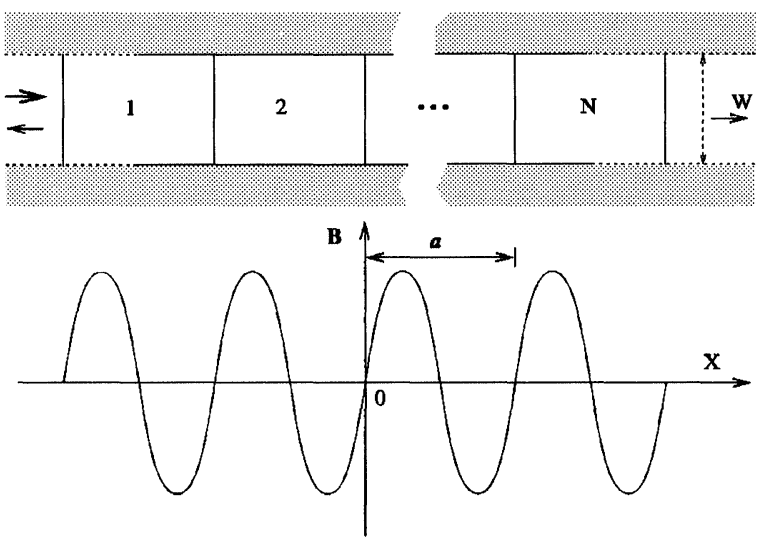

FIGURE 1 Schematic view of a quantum wire of width $W$ with hard-wall confinement in the transverse direction. A finite section is subject to a periodically varying magnetic field, with profile shown in the lower graph.

of $2 e^{2} / h$, due to propagation of a set of discrete modes through the wire. As the magnetic modulation is turned on, the transport will be affected by the oscillations of $\boldsymbol{B}(x)$, so some change in the character of the conductance is expected. We show in Figure 2 the conductance of the quantum wire as a function of $k_{F} W / \pi$ for $N=3, B_{m}=1.2 T$ and $B_{0}=0.3 T$, respectively. In the case of weak modulation, the conductance still shows a staircase structure with plateaus very close to the quantized values $2 e^{2} n / h(n$ being the number of subbands below $E_{F}$ ) as shown in Figure 2(a). Notice that there are dips in conductance just below the threshold of each mode. Similar dips have also been found in the conductance of perturbed quantum wires subjected to local potential disorder, or a potential barrier, or coupled to a resonant cavity, rather than magnetic modulation. Tekman and Bagwell [7] and Berthod et al. [8] have argued that sub-threshold dips like these are Fano resonances; [9] that is, they are due to coupling between a discrete state and the continuum in which it is situated. They have shown how the nearby threshold can induce a discrete bound or anti-bound state and so give rise to the dip.

The coupling between localized states and propagating modes can be revealed by the local current distribution. In the non-magnetic case, it

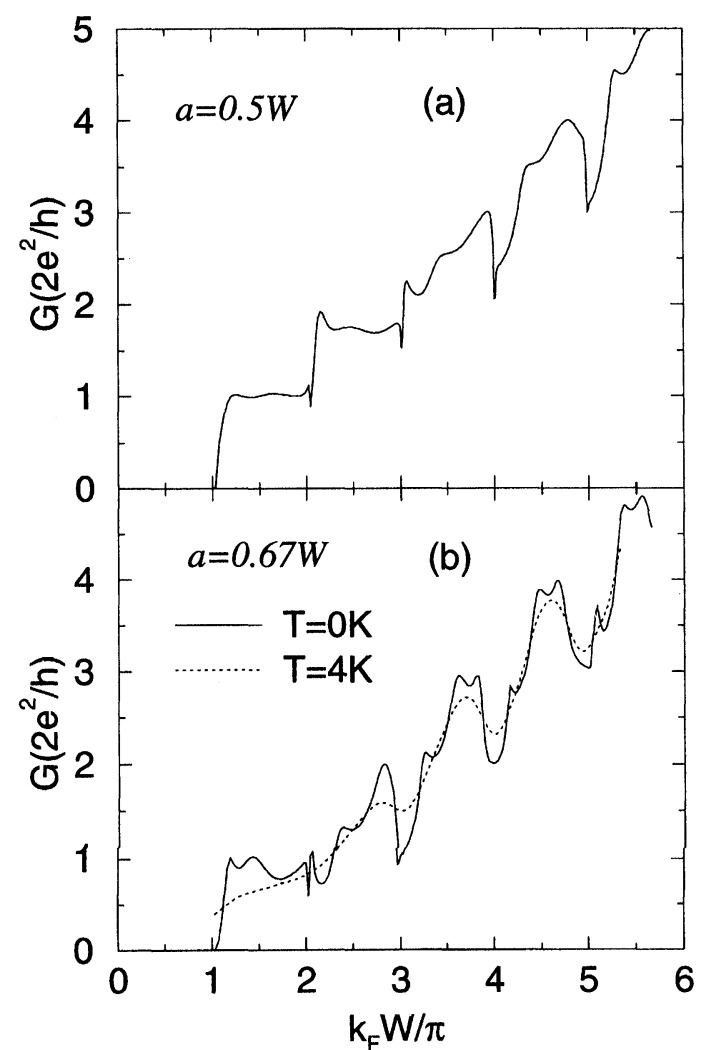

FIGURE 2 Conductance as a function of $k_{F} W / \pi$ for $N=3$, $B_{m}=1.2 T$ and $B_{0} 0.3 T$. (a) and (b) correspond to $a=0.5 \mathrm{~W}$ and $0.67 W$, respectively.

has been found that vortices in the current flow reverse their sense of rotation as the energy is raised through a resonance level $[8,10]$. We looked at current flows at a number of energies around the first conductance dip in Figure 2(a). Away from the dip, the flow is laminar on either side. In a narrow energy window, close to the dip, a number of vortices appear, indicating the presence of a localized state. In our example, with three periods of oscillation, there was one large vortex in each period. These move a little as the energy is raised, but the sense of rotation did not change. Rather, the dip was associated with one vortex moving out of the scattering region, leaving a current pattern transverse to the channel along the end of the scattering region, accounting for the reduced transmission. Since the three main vor- 
tices are counter-rotating, transmitted electrons follow a meandering path around them. The lack of reversal is understandable as these vortices are driven by the applied magnetic field.

When the background field $B_{0}$ is increased, the conductance dips shift to higher values of $k_{F} W / \pi$ due to the one-dimensional subbands in the leads, which gradually depopulate as an increasing, perpendicular magnetic field is applied (not shown here). In Figure 2(b) the length of the period $a$ is $0.67 \mathrm{~W}$. Lengthening the scattering region provides thicker magnetic barriers, which tends to reduce the transmission. We see that as $a$ increases, the conductance dips become more pronounced. We also get some conductance oscillations between the dips as we increase $a$; these are due to multiple reflections within the scattering region.

The temperature dependence of the conductance dips is shown in Figure 2(b). We find that the conductance dips are quite robust at low temperatures. The dotted line in Figure 2(b) shows the conductance at $T=4 \mathrm{Kelvin}$. The oscillations due to multiple reflections within the scattering region disappear, but the conductance dips are still apparent.

Figure 3 shows the conductance of a modulated quantum wire with $N=4$ and 8 unit cells, respectively. For a wire with $N=4$ unit cells the conductance shows an indication of miniband formation in a periodic structure. Although the basic features of the formation of minibands and gaps are already present for wires with a few unit cells, some narrow and fine minibands and gaps reveal themselves only for a rather long modulated wire, as seen in Figure 3 for the wire with $N=8$ periods.

In Figure 4 we show the calculated conductance of a wire with $N=16$ and the individual transmission coefficients for each incident mode. The conductance displays dips due to the formation of minigaps and rapid oscillations due to electron transmission through the coupled quasi-zerodimensional states in the cavity regions between the magnetic barriers. Each group of conductance oscillations evolves into a continuous miniband in

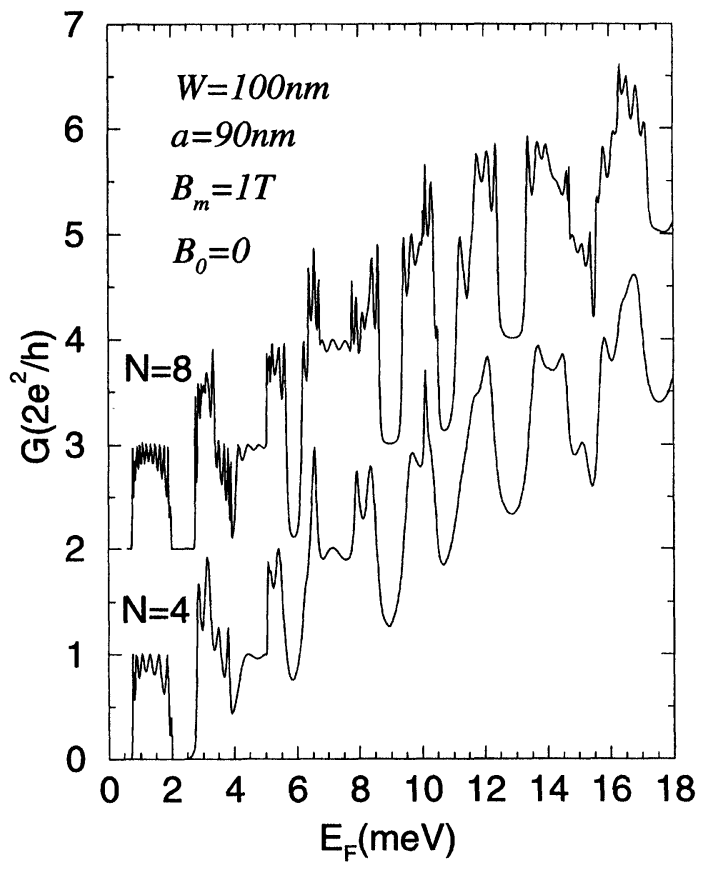

FIGURE 3 Conductance vs Fermi energy $E_{F}$ for quantum wires with $N=4$ and 8 , respectively. Curve for $N=8$ is vertically offset for clarity.

the limit of an infinitely long superlattice. The formation of minibands and minigaps in a finite lateral surface superlattice has been reported using split-gate structures, in which the 1D lateral surface superlattice is realized by a periodic modulation of constriction width instead of magnetic modulation [11].

In the electric modulated case, the number of oscillations in the first conduction plateau is equal to the number of cavity regions [12]. However, in our case the channel is modulated by the magnetic field. We can see that there is no simple direct correspondence between the number of unit cells and the number of oscillations. This result shows that the effective potential of the magnetic barriers for electron motion in the wire is complicated [13]. The oscillations in higher plateaus are more irregular because of the strong coupling between modes. The peak values of the conductance oscillations should be an integer multiple of $2 e^{2} / h$. Some of the peaks fail to reach a quantized 


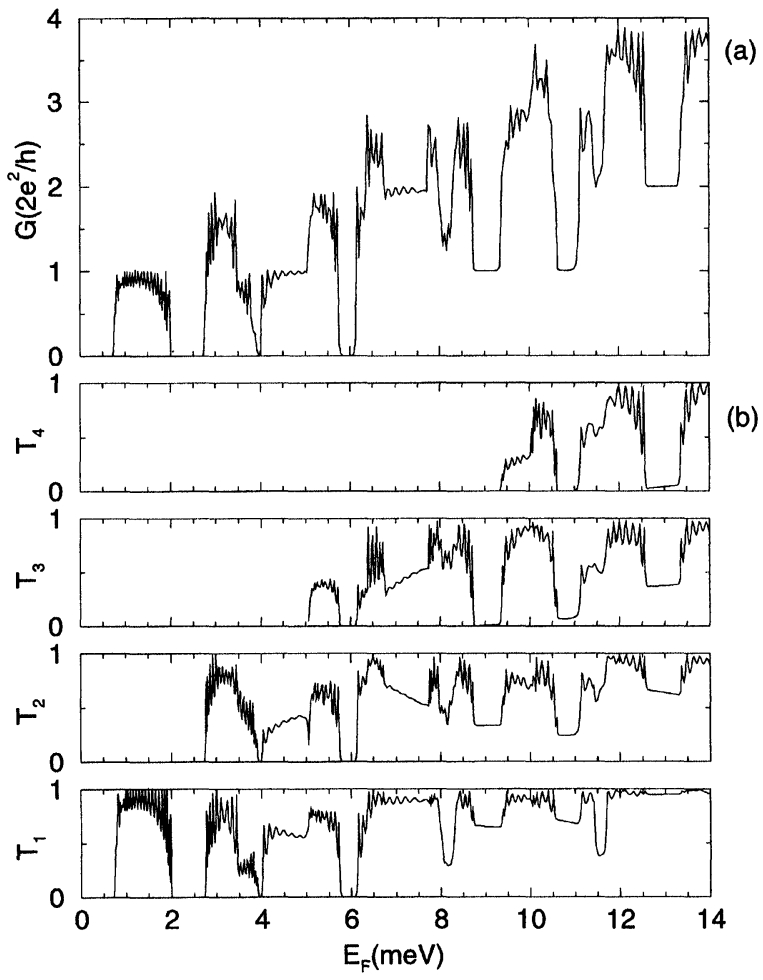

FIGURE 4 (a) Conductance as a function of Fermi energy $E_{F}$ for a finite period magnetic superlattice with 16 unit cells. $a=90 \mathrm{~nm}, W=100 \mathrm{~nm}, B_{0}=0$ and $B_{m}=1.5 \mathrm{~T}$. (b) Transmission coefficients of individual modes for the magnetic modulated quantum wire.

value due to the limited number of data points. The peaks are very narrow and therefore require more extensive computing. We find that the oscillations become more prominent as $a$ becomes longer. It is thus suggested that the formation of an effective loop of edge states is essential for the conductance oscillations as discussed by Takagaki [14] and Yoshioka [15]. One is then dealing with an Aharonov-Bohm type interference.

The conductance, plotted in Figure 4(a), steps up and down between quantized levels. Conductance quantization is related to the band structure for the corresponding infinite system [12]. However, none of the individual modal transmission coefficients in Figure 4(b) shows quantization by itself. See for example the region near $9 \mathrm{meV}$. The quantization occurs as the various modes are mixed by the periodic modulation.
The calculated conductance as a function of the amplitude $B_{m}$, is displayed in Figure 5(a). The conductance steps down by two-units of $2 e^{2} / h$ and up by one-unit, then down to zero as $B_{m}$ is increased. Transmission coefficients $T_{i}$ of indivi-
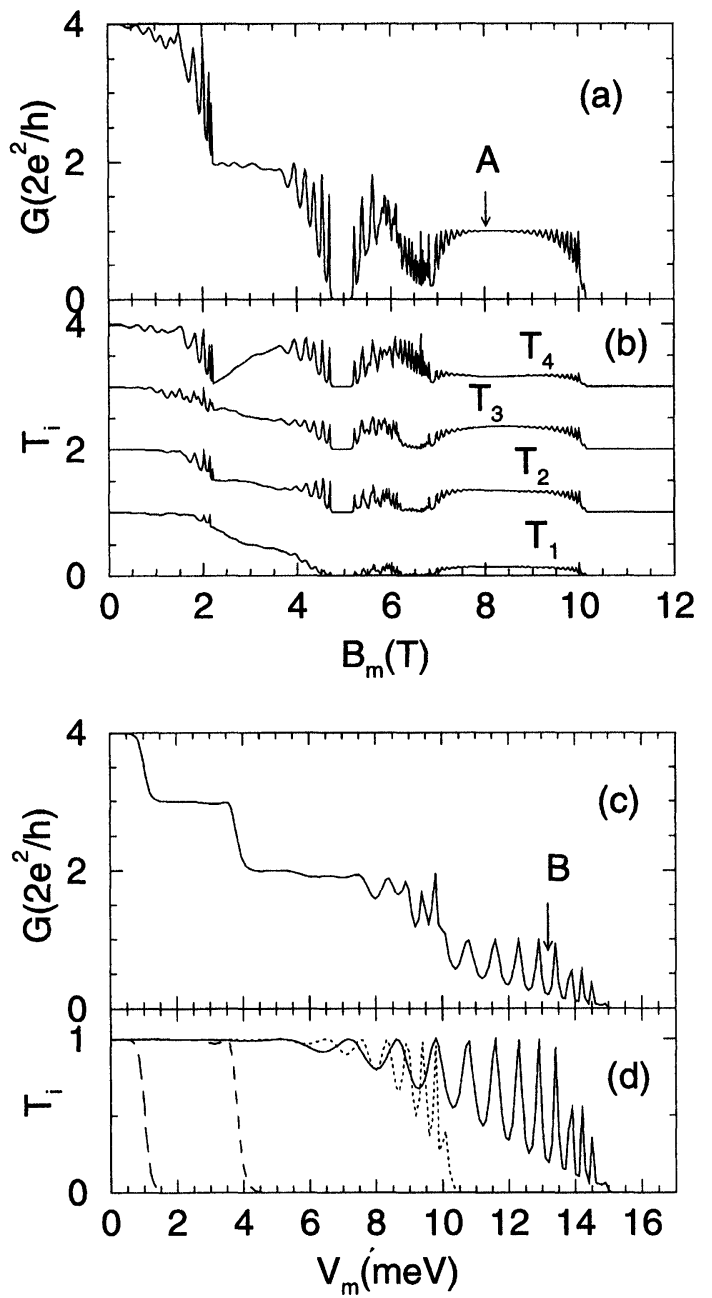

FIGURE 5 (a) The conductance as a function of the magnetic modulation amplitude $B_{m}$ for a quantum wire with 16 unit cells. $a=90 \mathrm{~nm}, W=100 \mathrm{~nm}, B_{0}=0$ and $E_{F}=12 \mathrm{meV}$. (b) Transmission coefficients $T_{i}$ of individual modes for the magnetic superlattice. The curves are offset for clarity. (c) The conductance as a function of the electric modulation amplitude $V_{m}$ for a finite period electric superlattice. The other parameters are the same as (a). (d) Transmission coefficients $T_{i}$ of individual modes for the electric superlattice. The solid, dotted, dashed and long-dashed curves correspond to $i=1,2,3$ and 4, respectively. The arrows A and B indicate the cases corresponding to the current distributions shown in Figures 6(a) and 6(b), respectively. 
dual modes for the magnetic superlattice are shown in Figure 5(b); the curves are vertically offset for clarity. Although the total conductance, which is the sum of the modal transmissions, is essentially quantized, the individual transmissions, plotted in Figure 5(b), show complicated dependence on $B_{m}$.

We also calculated the conductance for an electric superlattice with amplitude $V_{m}$ and sinusoidal barriers. The geometry of the structure is otherwise the same as that of Figure 5(a). The conductance for a 1D electric superlattice steps down monotonically as the propagating modes in the leads are blocked by the potential barriers with increasing electric modulation amplitude $V_{m}$, as shown in Figures 5(c) and 5(d). No two-unit drop is seen for increasing electric modulation amplitude $V_{m}$ in Figure 5(c). Clearly, the unique features of the wave-vector-dependent effective potential for the magnetic barriers are critical for the twounit drop in Figure 5(a). In our calculations, the two-unit drop in conductance with increasing $B_{m}$ is predominant but not universal; occasionally we find cases with a one-unit drop. The reason for this is that the effective potential of the magnetic barriers for electron motion along the quantum wire depends on the mode of the incident electron in the leads [13].

Figures 6(a) and 6(b) show the current densities in one unit cell of the magnetic/electric superlattices for the cases labelled by A and B in Figures 5(a) and 5(c), respectively. Figure 6(a) corresponds to the current pattern for the case that there is one propagating mode in the sample. The current pattern clearly shows that the Lorentz force allows electrons to move in a meandering way. At sufficiently high magnetic fields, the current flow displays a complex pattern. Figure $6(\mathrm{~b})$ shows the current distribution for an electric superlattice with sinusoidal barriers. Notice that in this case, the current flows straight across the cell. This illustrates that electron transport in this electric superlattice is equivalent to a one-dimensional system, in contrast to the magnetic one, in which it is not.

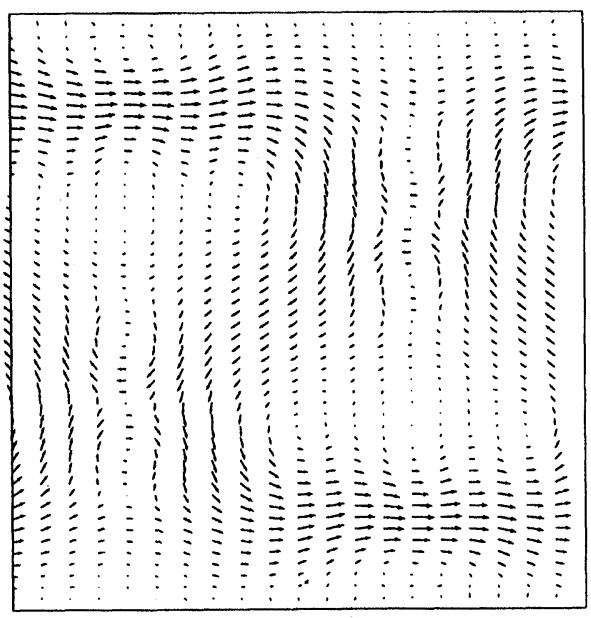

(a)

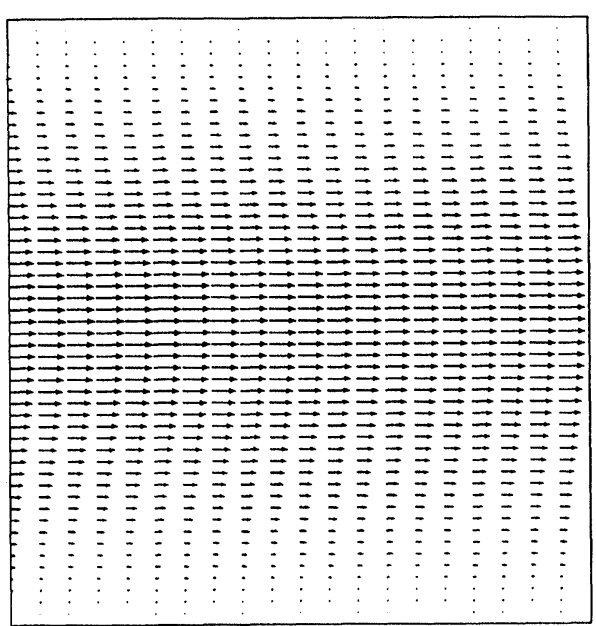

(b)

FIGURE 6 The current density distributions (a) and (b) corresponding to the cases labelled $\mathrm{A}$ and $\mathrm{B}$ indicated in Figures $5(\mathrm{a})$ and 5(c), respectively.

In conclusion, we have studied the transport properties of a quantum wire in spatially periodic magnetic and electrostatic fields. We found a conductance dip, an example of a Fano resonance, just below each mode threshold. These conductance dips shift to higher values of $k_{F} W / \pi$ when 
the background field is increased. As the length $a$ of the period increases, the conductance dips broaden and the dips for higher modes become more pronounced. The conductance dips are quite robust at low temperatures. We have shown that many features of electronic transport in 1D magnetic superlattices differ from those of the electrostatically modulated ones. We find that unlike the electric superlattice, the number of oscillations in the first conduction plateau has no simple direct correspondence to the number of unit cells $N$. The conductance of 1D magnetic superlattices does not decrease monotonically with increasing $B_{m}$ but rather steps up and down between quantized levels, sometimes going to zero. The spatial distributions of current density show dramatic differences between the magnetic and electric superlattices.

This complex behaviour suggests that the experimental study of 1D magnetic superlattices should be very interesting. Several methods are available to produce such devices, including the laying down of patterned superconducting strips on top of a gated device.

\section{Acknowledgements}

Work supported by NSERC research grant SAPOO-3198.

\section{References}

[1] Carmona, H. A., Geim, A. K., Nogaret, A., Main, P. C., Foster, T. J., Henini, M., Beaumont, S. P. and Blamire, M. G. (1995). "Two-dimensional Electrons in a Lateral Magnetic Superlattice", Phys. Rev. Lett., 74, 3009-3012.

[2] Ye, P. D., Weiss, D., Gerhardts, R. R., Seeger, M., von Klitzing, K., Eberl, K. and Nickel, H. (1995). "Electron in a Periodic Magnetic Field Induced by a Regular Array of Micromagnets", Phys. Rev. Lett., 74, 3013-3016.

[3] Izawa, S., Katsumoto, S., Endo, A. and Iye, Y. (1995). "Two-dimensional Electrons in Lateral Magnetic Superlattice", J. Phys. Soc. Jpn., 64, 706-710.

[4] Leadbeater, L., Foden, C. L., Burke, T. M., Burroughes, J. H., Grimshaw, M. P., Ritchie, D. A., Wang, L. L. and Pepper, M. (1995). "Electron Transport in a NonUniform Magnetic Field”, J. Phys. Condens. Matter, 7, L307-L315.
[5] Ji, Zhen-Li and Sprung, D. W. L. (1996). "Electronic Transport in Quantum Wires in Nonuniform Magnetic Fields", Phys. Rev. B, 54, 8044-8048.

[6] Ji, Zhen-Li and Sprung, D. W. L. (1997). "Transport Properties of Quantum Wires in Spatially Periodic Magnetic and Electrostatic Fields", Semicond. Sci. Technol., 12, 529-534.

[7] Tekman, E. and Bagwell, P. F. (1993). "Fano Resonances in Quasi-one-dimensional Electron Waveguides", Phys. Rev. B, 48, 2553-2559.

[8] Berthod, C., Gagel, F. and Maschke, K. (1994). "DC Transport in perturbed multichannel quantum wires", Phys. Rev. B, 50, 18299-18311.

[9] Fano, U. (1961). "Effects of Configuration Interaction on Intensities and Phase Shifts", Phys. Rev., 124, 1866-1870.

[10] Ji, Zhen-Li (1993). "Ballistic Transport through a Double Bend in an Electron Waveguide", J.'Appl. Phys., 73, $4468-4472$.

[11] Kouwenhoven, L. P., Hekking, F. W. J., van Wees, B. J., Harmans, C. J. P. M., Timmering, C. E. and Foxon, C. T. (1990). "Transport through a Finite One-dimensional Crystal", Phys. Rev. Lett., 65, 361-364.

[12] Leng, M. and Lent, C. S. (1993). "Recovery of Quantized Ballistic Conductance in a Periodically Modulated Channel", Phys. Rev. Lett. 71, 137-140.

[13] Ibrahim, I. S. and Peeters, F. M. (1995). "Two-dimensional Electrons in Lateral Magnetic Superlattices", Phys. Rev. B, 52, 17321-17334.

[14] Takagaki, Y. and Ploog, K. (1995). "Tunneling Transmission in Quantum Wires in the Presence of Magnetic Fields", Phys. Rev. B, 51, 7017-7023.

[15] Yoshioka, H. and Nagaoka, Y. (1990). "Effect of Magnetic Field on the Conductance of Ballistic Quantum Wire", J. Phys. Soc. Jpn., 59, 2884-2892.

\section{Authors' Biographies}

Zhen-Li Ji received a Licentiate of Engineering degree in 1991 and a Ph.D. degree in 1993 from Linköping University. He remained at Linköping for two years of postdoctoral research experience. $\mathrm{He}$ is currently working in the Dept. of Physics and Astronomy, McMaster University as a postdoctoral fellow. His research interests include electronic transport properties in low-dimensional semiconductor nanostructures, such as ballistic transport, resonant tunnelling, conductance fluctuations, chaotic behaviour, impurities, geometrical effects, quantum Hall effect and nonlinear transport in semiconductor nanostructures.

D.W.L. Sprung received a Ph.D. in Mathematical Physics in 1961 from the University of Birmingham, with R. E. Peierls. Following a post-doctoral year with $\mathrm{H}$. A. Bethe at Cornell he joined the faculty of McMaster University 
where he has chaired the Departments of Physics and Astronomy and Applied Mathematics and has served as Dean of the Faculty of Science for two terms. His research interests have ranged over nuclear structure theory, few-body problems and quantum nanostructures. 

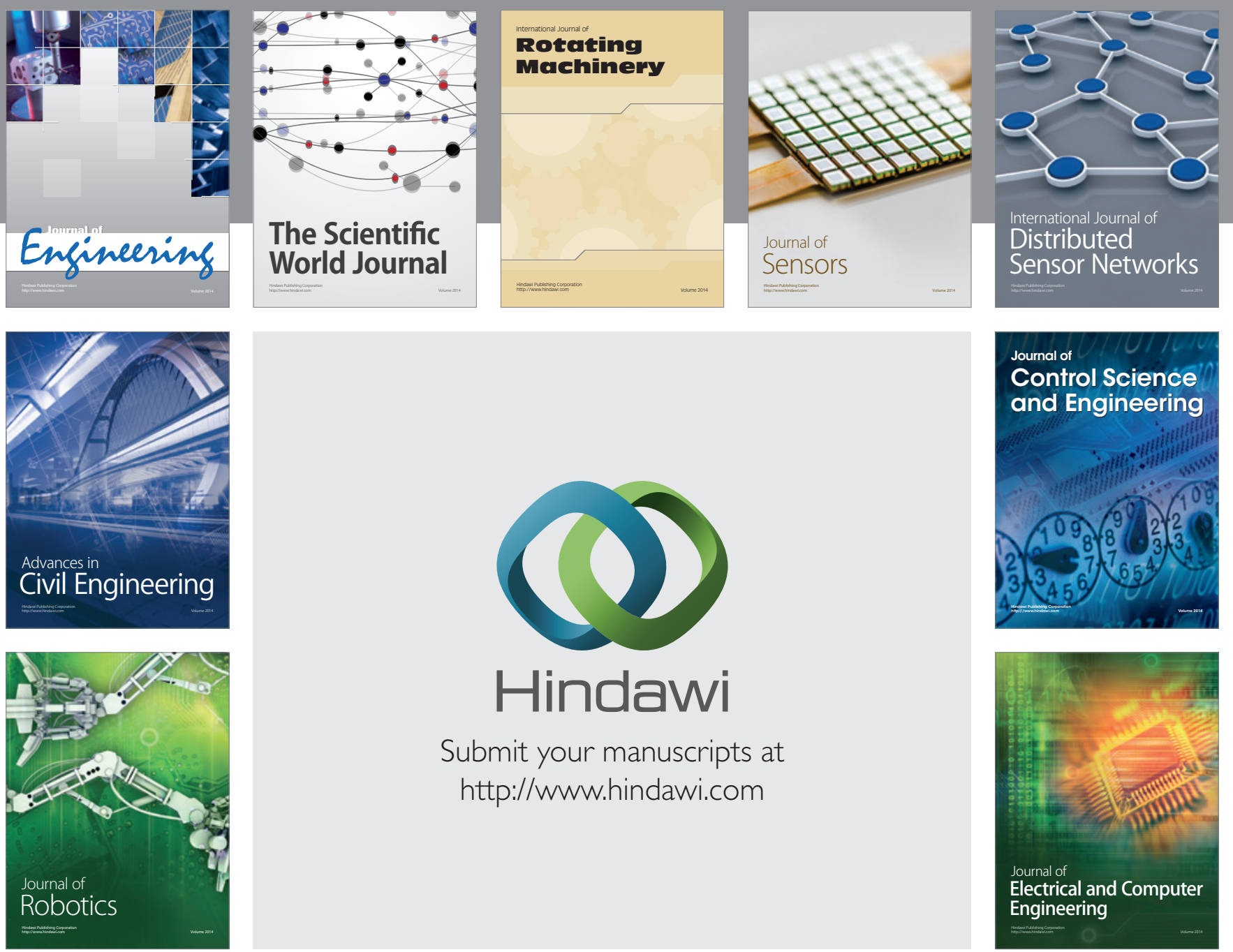

Submit your manuscripts at

http://www.hindawi.com
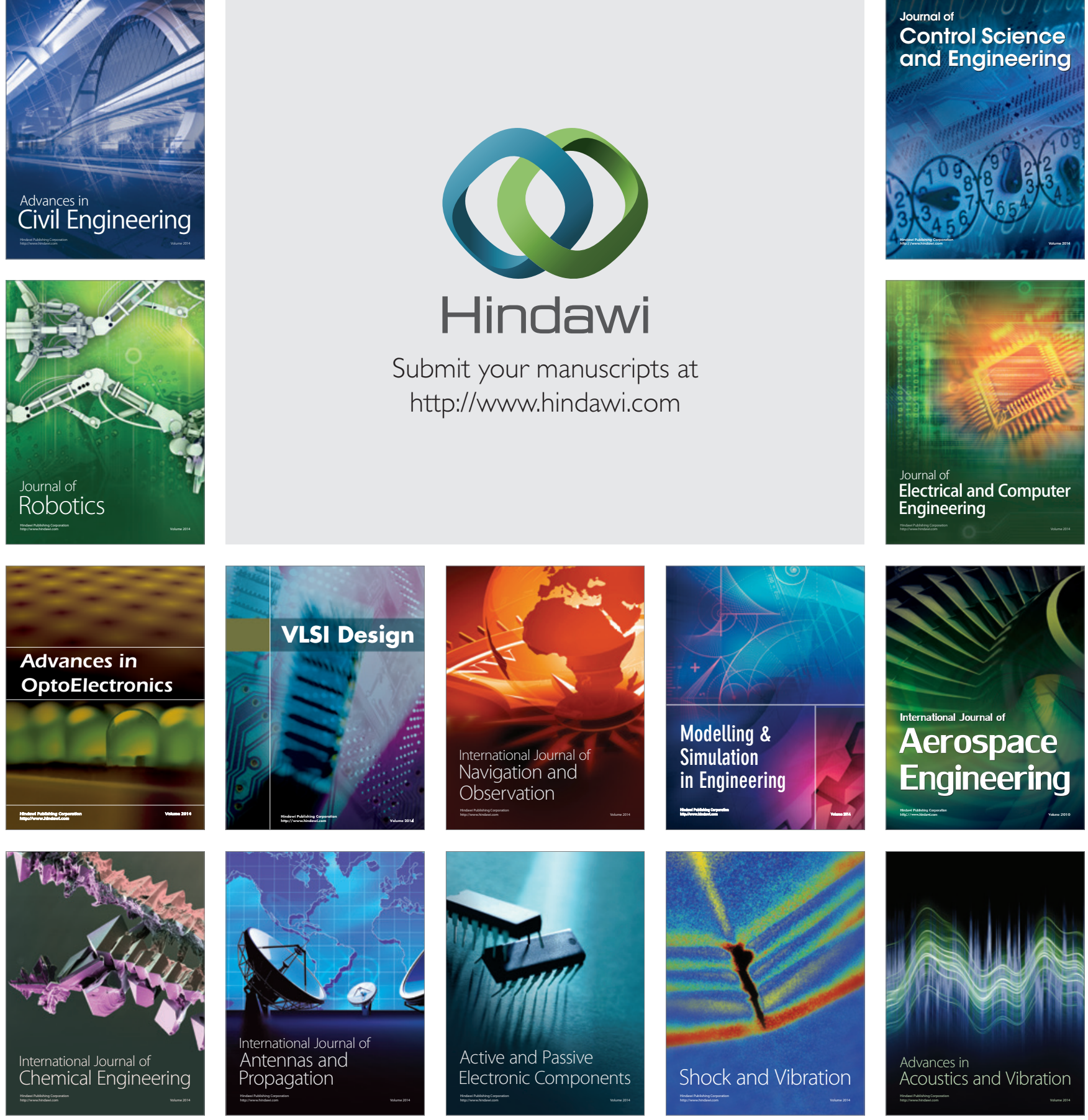